\title{
Control of core argon impurity profile by ECH in KSTAR L- mode plasmas
}

\author{
Joohwan Hong ${ }^{1}$, Seung Hun Lee ${ }^{1}$, Juhyung Kim², C. R. Seon², S. G. Lee², G. Y. Park ${ }^{2}$, K. D. \\ Lee $^{2}$, S. S. Henderson ${ }^{3}$, H. Y. Lee ${ }^{1}$, Jae Sun Park ${ }^{1}$, Juhyeok Jang ${ }^{1}$, Siwon Jang ${ }^{1}$, Taemin Jeon ${ }^{1}$, \\ M. O’Mullane ${ }^{3}$, and Wonho Choe ${ }^{1, *}$ \\ ${ }^{1}$ Department of Physics, Korea Advanced Institute of Science and Technology, 291 Daehak-ro, Yuseong-gu, \\ Daejeon, Republic of Korea \\ ${ }^{2}$ National Fusion Research Institute, 169-148 Gwahak-ro, Yuseong-gu, Daejeon, Republic of Korea \\ ${ }^{3}$ Department of Physics, University of Strathclyde, 16 Richmond Street, Glasgow G1 1XQ. Scotland, United \\ Kingdom \\ *Author to whom corresponding should be addressed; E-mail: wchoe@kaist.ac.kr
}

\begin{abstract}
Experiments on trace argon impurity transport in L-mode discharges were performed on Korea Superconducting Tokamak Advanced Research (KSTAR) with electron cyclotron resonance heating (ECH). Ar emission was measured by soft X-ray (SXR) arrays and vacuum UV (VUV) diagnostics. A significant reduction in the core Ar emissivity was observed with core $\mathrm{ECH}$. The reduction was the largest with on-axis heating and became smaller with outward heating positions. The diffusivity and convection velocity of Arwere obtained by analysis of the SXR data with the SANCO impurity transport code for the on-axis ECH and the non-ECH shots. In the on-axis ECH case, both diffusivity and convection velocity increased. Furthermore, the convection changed its direction from inward to outward in the plasma core $(\mathrm{r} / \mathrm{a}<0.3)$, resulting in a hollow profile of the total Ar density. Together with the reduction in the SXR signals, the hollow impurity profile in the core and the reversal of the convection velocity consistently confirm that $\mathrm{ECH}$ can reduce impurity accumulation in the core region. Neoclassical impurity transport and linear stability of micro-turbulence were calculated and discussed in relation to the possible transport mechanism.
\end{abstract}




\section{Introduction}

Controlling impurities inside the plasma is an urgent and critical issue for ITER [1] and future fusion reactors. Impurities produce detrimental effects on high-performance tokamak operations by diluting the main fuel ions and causing significant radiation cooling [2], especially in the plasma core via line radiation and continuum radiation, such as bremsstrahlung and recombination radiation [3]. Therefore, understanding and finding ways to control impurity accumulation [4] inside the plasma have received much attention in the fusion community. Use of auxiliary heating, such as electron cyclotron heating $(\mathrm{ECH})$ and ion cyclotron resonance heating (ICRH), has been attempted in various devices in order to avoid core accumulation. Many experiments with those heating tools have demonstrated a reduction in core accumulation with enhanced impurity transport [5-13]. On the theoretical side, neoclassical transport theories have yet to explain many experimental results regarding impurity transport. There has been recent progress in exploring possible mechanisms for turbulence-driven impurity transport $[14,15]$ and neoclassical transport including the centrifugal effects for heavy impurity ions $[16,17]$. Here, we provide the measurements and analysis of argon impurity transport in KSTAR in an effort to clarify the nature of impurity transport. Experiments to investigate impurity transport, particularly in superconducting tokamaks such as KSTAR, would be timely and important for upcoming ITER operations.

In this paper, experiments on Ar impurity transport on KSTAR are presented. In the experiments, L-mode discharges are additionally heated by ECH with trace Ar injected by gas puffing at the plasma current flattop phase. In sections 2 and 3, the experimental setup and diagnostics for measuring Ar emission and the raw data are described. Then, the analysis of Ar transport coefficients by SANCO is presented in section 4, which is followed by discussion and summary in sections 5 and 6 .

\section{Diagnostics and s hot de scription}

A set of Ar Ross filters was installed in the multi-channel soft X-ray (SXR) array diagnostic system [18] to investigate transient Ar transport phenomena. The Ar Ross filters are made of sodium chloride $(\mathrm{NaCl})$ and calcium fluoride $\left(\mathrm{CaF}_{2}\right)$ whose absorption edges are $2.8224 \mathrm{keV}$ and $4.0385 \mathrm{keV}$, respectively [19-21]. Each transmittance curve combined with a Si photodiode detector is shown in Ref. [18] as a function of incident photon energy. Adjusting the thickness of the filters can balance out the $\mathrm{X}$-ray signals outside the range between the two K-edges. The Ar Ross filters pass soft X-ray photons in the energy range between the two K-edges containing the line emissions of $\mathrm{Ar}^{16+}$ and $\mathrm{Ar}^{17+}$. The $\mathrm{X}$ ray detector consists of $16 \mathrm{Si}$ photodiodes (Opto Diode Corp. AXUV16-ELG) aligned side by side, and each photodiode is assigned for one viewing chord. Figure 1 shows a cross-sectional view of KSTAR and viewing chords. The up and down SXR arrays have two detectors, respectively, which are placed right next to each other. Because the distance between the two detectors $(30 \mathrm{~mm})$ is negligible compared with the length of the line of sight $(1.3 \mathrm{~m})$, the two detectors share the same views of the plasma volume, resulting in 16 viewing chords for each of the up and down arrays. 


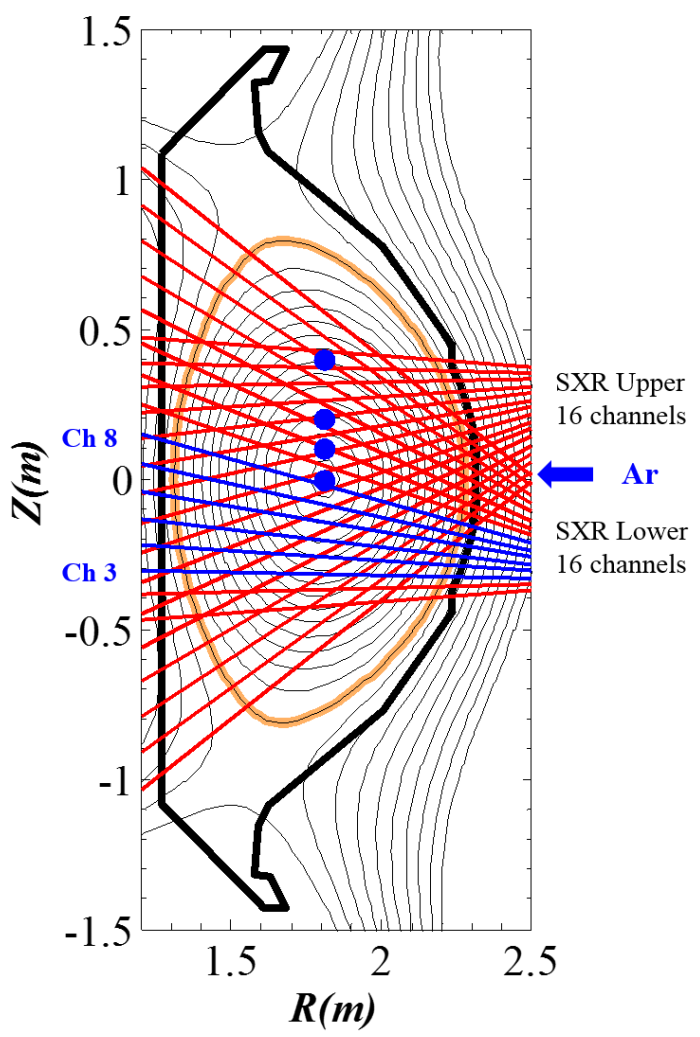

Figure 1. Line of sight of the up and down SXR channels. The blue dots in the figure indicate ECH positions corresponding to $\mathrm{r} / \mathrm{a}=0,0.16,0.30$, and 0.59 , respectively. The Ar gas was puffed from the midplane.

Experiments on trace argon impurity transport in the KSTAR L-mode discharges $\left(I_{p}=400 \mathrm{kA}, B_{T}\right.$ $=2 \mathrm{~T}$ ) were performed to analyze the local effect of $\mathrm{ECH}$ on the Ar impurity transport and core accumulation. Four different cases of $\mathrm{ECH}$ with different heating positions were compared with the reference case of non-ECH. We applied ECH with the maximum power of $350 \mathrm{~kW}$ and duration of 0.7 $\mathrm{s}$. The position of $\mathrm{ECH}$ was changed by tilting the focusing mirror along the vertical coordinate $z$. The heating positions of $z=0,0.1 \mathrm{~m}, 0.2 \mathrm{~m}$, and $0.4 \mathrm{~m}$, corresponding to $r / a=0,0.16,0.30$, and 0.59 , were chosen, where $r$ is the minor radial coordinate, and $a$ is the minor radius. The heating positions are denoted as blue dots in figure 1. For each case, the same amount of Ar gas was injected at the plasma current flattop phase of the discharges by a gas puffer installed at the mid-plane. It was injected for 20 $\mathrm{ms}$, and the total number of Ar atoms estimated by a calibrated gas flow meter was approximately $10^{17}$ atoms. This is a trace amount of impurities that perturbs the electron temperature and density by less than $10 \%$, as shown in figure 2. ECH was applied at $0.1 \mathrm{~s}$ before Ar injection. The details of the discharges are summarized in table 1 . Shot \#7566 is the case without ECH (non-ECH), and \#7574, $\# 7636, \# 7597$, and \#7610 are $4 \mathrm{ECH}$ shots with resonance layer positions at $r / a=0,0.16,0.30$, and 0.59 , respectively.

In addition to the line emission by Ar impurities, continuum radiation due to other ions may contribute to the soft X-ray signals with Ar Ross filters. The continuum radiation from electrons and other ions was subtracted by using reference shots without Ar gas puffing. As shown in figure 2, the non-Ar-puffing shot and the Ar-puffing shot have almost the same background plasma parameters with, at most, a $5 \%$ difference. 
(a)

(b)

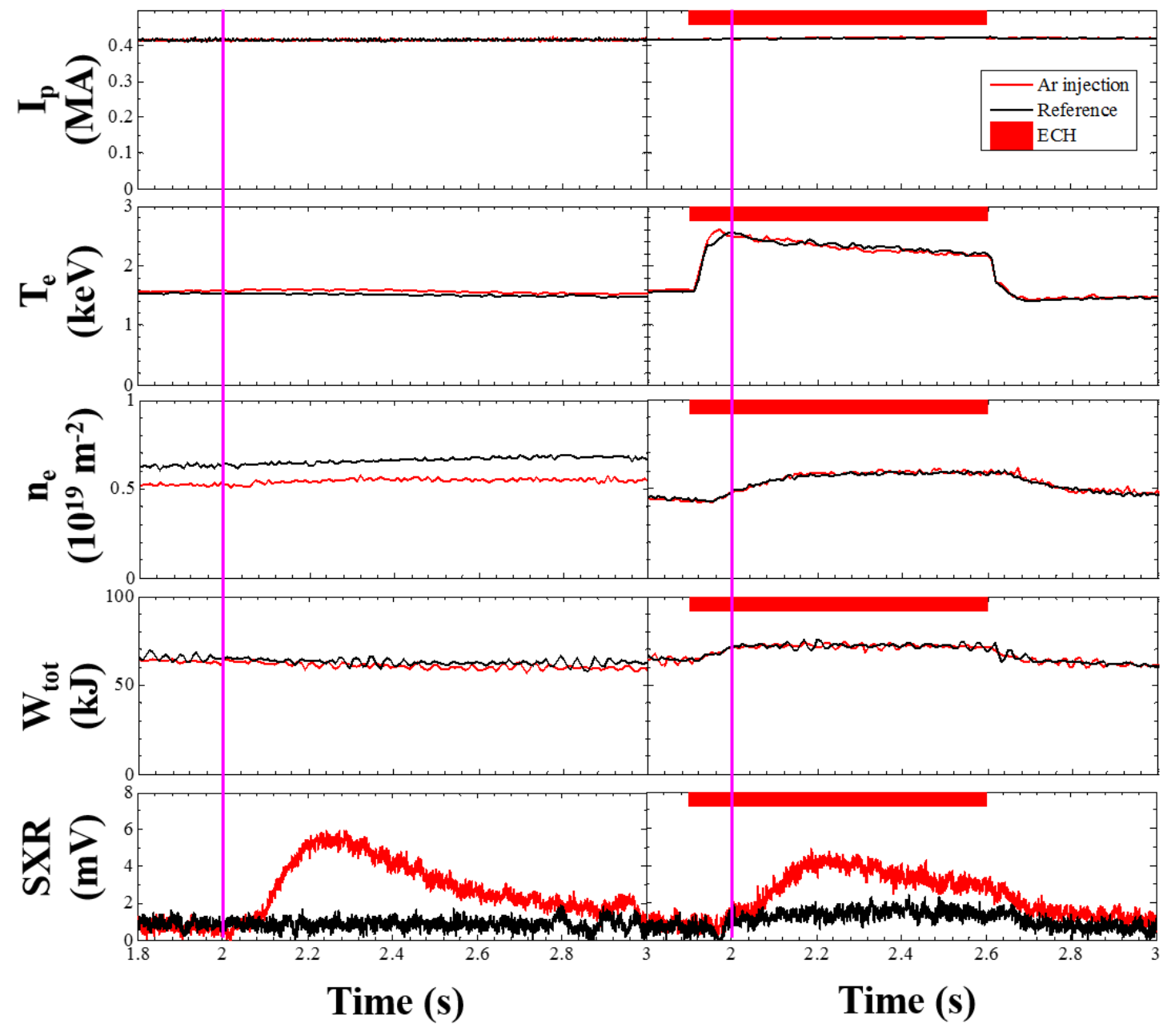

Figure 2. Main plasma parameters of (a) non-ECH and (b) on-axis ECH: plasma current $\mathrm{I}_{\mathrm{p}}$, electron temperature $T_{e}$, line-integrated electron density $n_{e}$, stored energy $W_{\text {tot }}$, and normalized central soft Xray chord signal. The black and red curves represent the non-puffing shot and Ar-puffing shot signals, respectively. The plasma conditions are similar to each other except the Ar impurity signals.

Table 1. Description of the experimental conditions. Electron density and temperature are averaged between $2-2.25 \mathrm{~s}$ (yellow box in figure 3(b)) for each shot.

\begin{tabular}{ccccc}
\hline Shot \# & $\begin{array}{c}J n_{e} d l \\
\left(10^{19} \mathrm{~m}^{-2}\right)\end{array}$ & $T_{e 0}(\mathrm{keV})$ & $\begin{array}{c}\mathrm{ECH} \\
(\mathrm{kW}, r / a)\end{array}$ & $\begin{array}{c}\text { Heating } \\
\text { duration }(\mathrm{s})\end{array}$ \\
\hline$\# 7566$ & 0.53 & 1.66 & - & - \\
$\# 7574$ & 0.58 & 2.31 & 350,0 & 0.7 \\
$\# 7636$ & 0.60 & 2.13 & $300,0.16$ & 0.4 \\
$\# 7597$ & 0.67 & 1.89 & $350,0.30$ & 0.7 \\
$\# 7610$ & 0.73 & 1.72 & $350,0.59$ & 0.7 \\
\hline
\end{tabular}

\section{Measured two-dime nsional argon emissivities with and without ECH}


The time evolutions of the central soft X-ray chord signals are shown in the bottom plot of figure 2 without [figure 2(a)] and with on-axis ECH [figure 2(b)]. Each plot includes non-Ar-puffing (black curve) and Ar-puffing cases (red curve). The soft X-ray signal starts to increase after the Ar gas is injected at $2 \mathrm{~s}$. The Ar-puffing signal contains an offset voltage due to the bremsstrahlung and recombination continuum radiation between the $\mathrm{Cl} \mathrm{K}$-edge and the Ca K-edge [18]. This offset signal before Ar injection is almost the same as the non-puffing signal. The subtraction of the non-puffing signal from the Ar-puffing signal represents mostly $\mathrm{Ar}^{16+}$ and $\mathrm{Ar}^{17+}$ line radiation. As shown in figure 2, the Ar emissivity of the on-axis ECH case is lower by a factor of 0.6 than that of the non-ECH case, suggesting that the on-axis ECH prevents the core accumulation of Ar ions.

The time evolution of the emissivity of the soft X-ray channels is plotted in figure 3(a). The dotted line in each plot indicates the line of sight passing through the plasma core, and the direction vertically away from the dotted line denotes the minor-radial coordinate. The soft X-ray signals of the non-ECH shot and the ECH shots with various heating positions are depicted from top to bottom in figure 3(a). For the sake of comparison, the emission intensities are normalized by the electron density of each shot since the emissivity of atomic Ar radiation is proportional to electron density. Reduction in the core impurity emissivity (see the color along the dotted line of each plot) is shown with ECH. As demonstrated in figure 3(b) where the soft X-ray signals of each central chord are plotted, the ECH effect of reducing the Ar signal is the strongest with on-axis heating and becomes weaker with outward heating positions. The peak signal increases as the ECH position moves radially outward and is the highest without ECH. The ECH effect is particularly significant when the deposition is inside $r / a=0.3$. The time trace of the core SXR signals that are normalized separately for each shot is shown in figure $3(\mathrm{c})$. It is noted that a higher diffusivity should translate in earlier peaks.

\section{Argon transport coefficients}

The diffusion coefficient and convection velocity of the impurity ions were determined by matching of the measured soft X-ray signals with the modeled emission using the Stand Alone NonCorona (SANCO) impurity transport code [22]. It solves the continuity equations for the impurity ions with each charge state $Z$ :

$$
\frac{\partial n_{Z}(r, t)}{\partial t}=-\nabla \cdot \Gamma_{Z}(r, t)+S_{Z}(r, t)
$$

where $n_{Z}$ and $\Gamma_{Z}$ are the density and the particle flux of the impurity ions, respectively. The source and $\operatorname{sink} S_{Z}$ of the impurities include electron impact ionization, electron recombination. All atomic data for the above processes are provided by ADAS [23]. A simple edge model [24] solving the parallel loss of particles between the last closed flux surface and the scrape-off layer is included in $S_{Z}$ as well.

The ansatz for particle flux is defined by a diffusion coefficient $D(r, t)$ and a convection velocity $V(r, t)$ as

$$
\Gamma_{Z}(r, t)=-D(r, t) \frac{\partial n_{z}(r, t)}{\partial r}+V(r, t) n_{z}(r, t)
$$

From equation (2) with given transport coefficients $D(r, t)$ and $V(r, t)$, the temporal evolution of $n_{Z}(r, t)$ as well as the total number of impurity ions $n_{A r}(r, t)=\sum_{z} n_{Z}(r, t)$ inside the last closed flux surface were calculated. In this study, the background electron temperature profiles were obtained from 
(a)

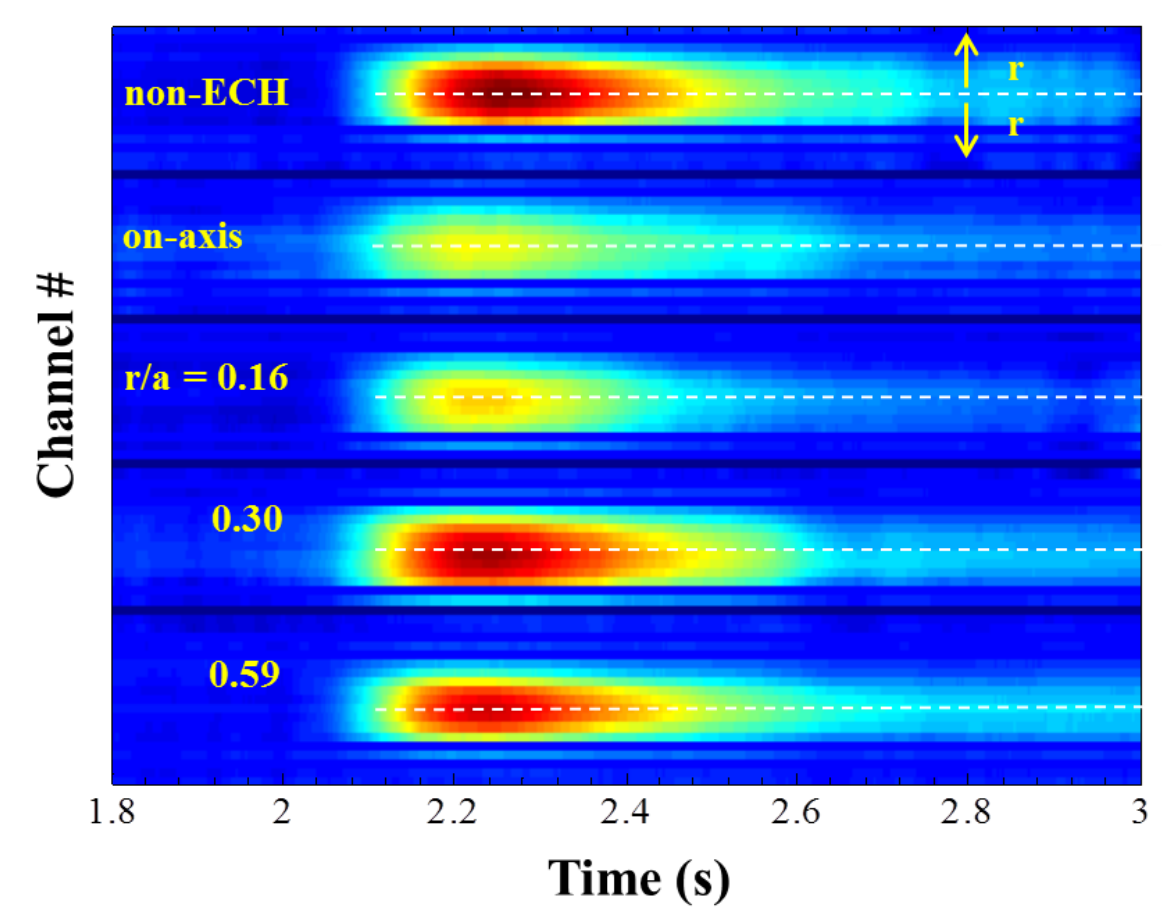

(b)

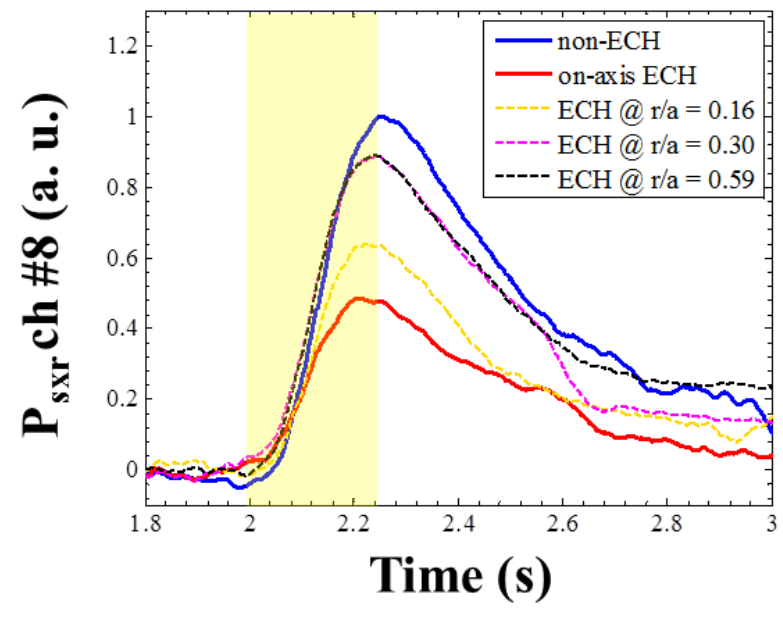

(c)

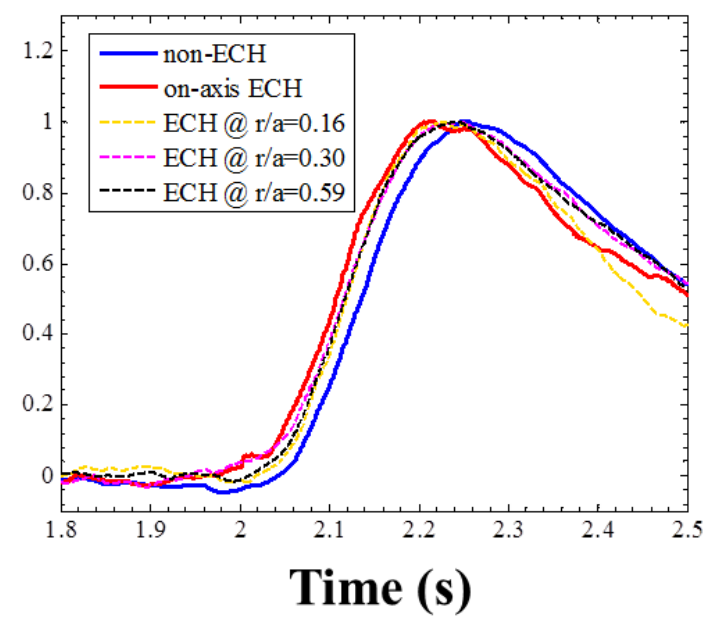

Figure 3. (a) Soft X-ray signal due to Ar impurity injection in various ECH conditions normalized by $\mathrm{n}_{\mathrm{e}}$. (b) Time trace of the core SXR signals. The yellow box indicates Ar influx phase under SANCO analysis. (c) Time trace of the core SXR signals that are normalized separately for each shot.

the electron cyclotron emission diagnostics. The electron density $\left(n_{e}\right)$ profile used for the Ar transport study was constructed by the 5-D drift kinetic XGC0 code [25] with the typical anomalous diffusion profiles of electron and ECE $T_{e}$ profiles as an input. The total number of electrons was verified by using the line-integrated electron density from the interferometer measurement. Figure 4 shows the radial profiles of electron density, electron temperature, and ion temperature for non-ECH and on-axis $\mathrm{ECH}$ cases. All the profiles were averaged in time between $2.00-2.25 \mathrm{~s}$ under the analysis. As for $\mathrm{T}_{\mathrm{i}}$, we used the charge exchange spectroscopy $\mathrm{T}_{i}$ profiles from the L-mode shots (\#8892, \#8896) that had the same parameters as our Ar injection experiment. 
(a) $\mathbf{n}_{\mathrm{e}}\left(10^{19} / \mathrm{m}^{3}\right)$

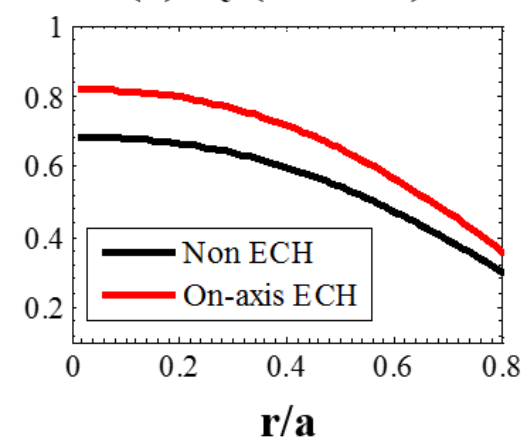

(b) $\mathrm{T}_{\mathrm{e}}(\mathrm{keV})$

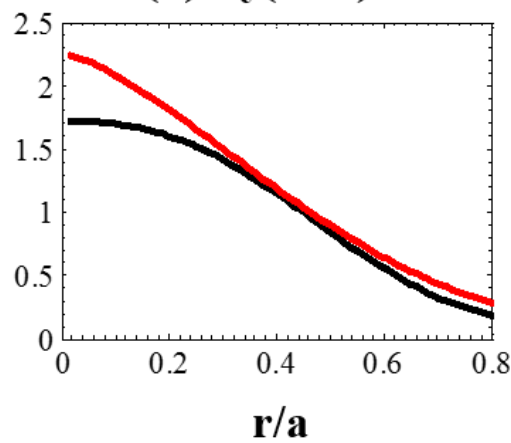

(c) $T_{i}(\mathrm{keV})$

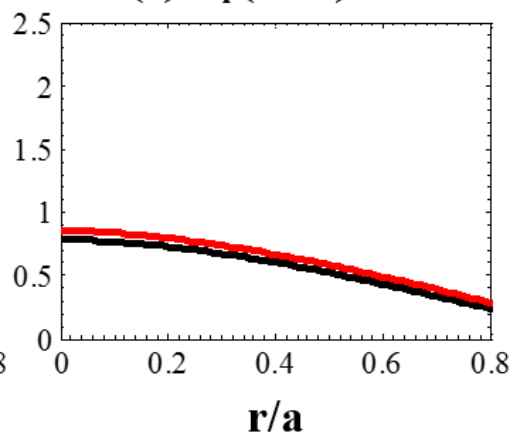

Figure 4. The radial profiles of (a) electron density, (b) electron temperature, and (c) ion temperature for the simulations of non-ECH and on-axis ECH cases.

The transport coefficients were considered to be time independent because the main parameters of the plasma were more or less constant during the time period under analysis as shown in figure 2 . After calculation of $n_{Z}(r, t)$, the local Ar radiation power $P_{A r}$ from the soft X-ray arrays is modeled by

$$
P_{A r}=\sum_{z=0}^{17} n_{e}(r, t)\left\{n_{z}(r, t) P_{L T}\left(T_{e}, n_{e}\right)+n_{z+1}(r, t) P_{R B}\left(T_{e}, n_{e}\right)\right\}
$$

where $n_{e}$ is the electron density, and $P_{L T}$ and $P_{R B}$ are the power coefficients from line emission driven by collisional excitation and from bremsstrahlung and recombination, respectively. Both $P_{L T}$ and $P_{R B}$ are calculated by ADAS using the transmission function of the Ross filter in the energy band of $2.8-4.0$ $\mathrm{keV}$. The total Ar radiation power is obtained by integrating the local radiation power along the line of sight of each channel. The modeled Ar radiations are then matched with the measured soft X-ray emissivities by adjustment of $D(r)$ and $V(r)$ until the minimum $\chi^{2}$ is found by the fitting analysis code, the Universal Transport Code (UTC) [26], which is implemented in SANCO. It is noted that the fit is well matched in the absolute value and the unit of $\mathrm{W} / \mathrm{m}^{2}$, not in an arbitrary unit without any normalization of raw data or simulation data. Among the Ar injection shots, the non-ECH plasma (\#7566) and the on-axis ECH plasma (\#7574) were analyzed. The EFIT magnetic equilibrium profiles were used as computational grids for the line-of-sight and flux averaging calculation, and the measured Ar influx from the Ar gas flow meter were used as an input for the SANCO calculation.

Since we focused on the core accumulation and peak density distribution of Ar, the time between $2 \mathrm{~s}$ (right after Ar puffing) and $2.25 \mathrm{~s}$ (right before decaying of the Ar emission) were chosen for the transport calculation, which is the Ar influx phase and is highlighted in the shaded box in figure 3(b). The input background plasma parameters were averaged in the influx phase. The best fits of the modeled Ar radiation to the soft X-ray data for the non-ECH (\#7566) and the on-axis ECH (\#7574) cases are shown in figure 5(a) and (b), respectively. The SANCO simulations (denoted by red) matched well with the soft X-ray data (denoted by black dots with error bars). The corresponding $D(r)$ and $V(r)$ are plotted in figure 6 for both non-ECH and ECH cases, respectively. The gray-shades in $r / a>0.5$ in the figure indicate high uncertainty due to the low signal-to-noise ratio in the soft X-ray signals, particularly from the edge channels. 
(a)

Ch $3(r / a=0.5) \quad$ Ch $4(r / a=0.42) \quad$ Ch $5(r / a=0.35)$

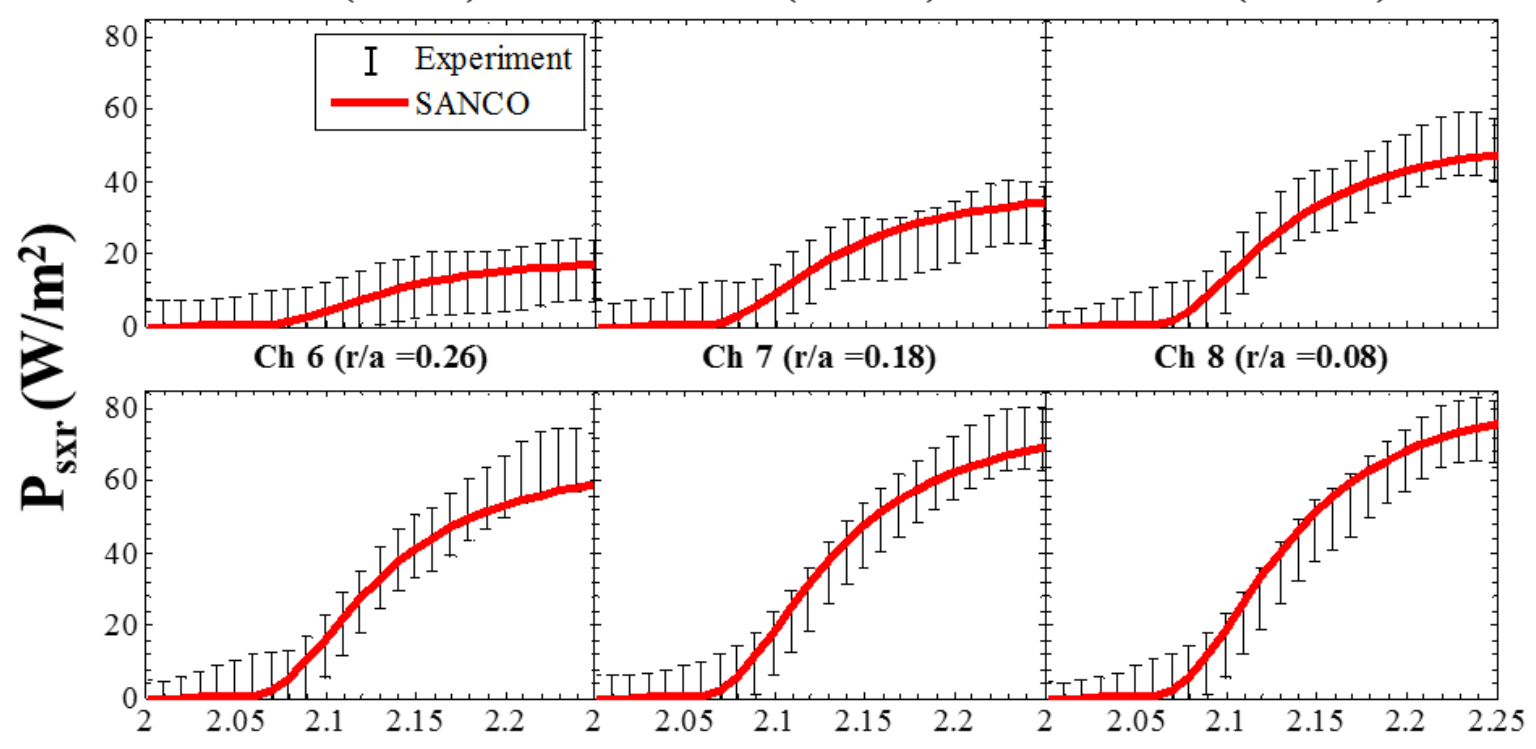

(b)
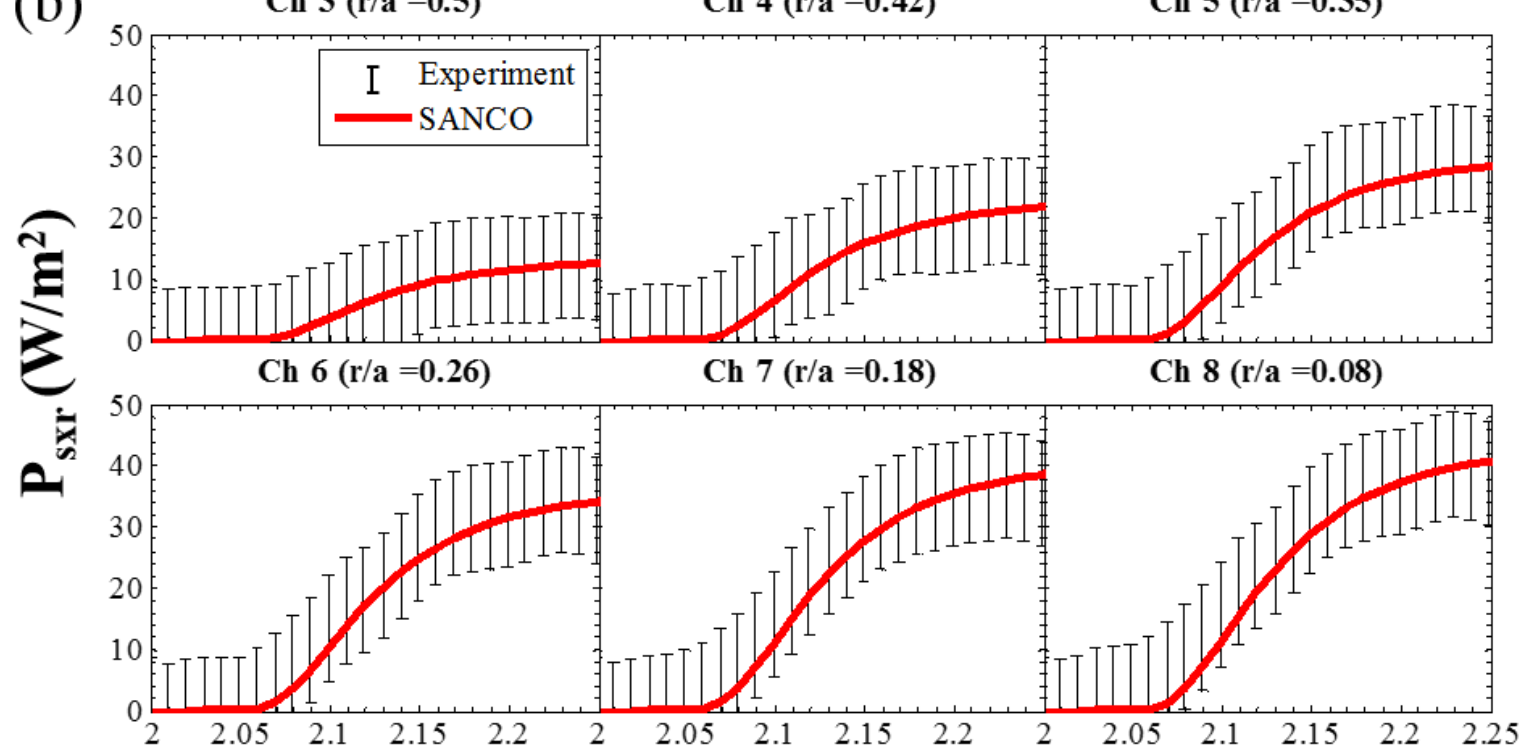

Time (s)

Figure 5. Red curves are SANCO simulation result, and black dots with error bars indicate Ar emissivities in power $\left(\mathrm{W} / \mathrm{m}^{2}\right.$ ) for the (a) non-ECH case (\#7566) and (b) on-axis ECH case (\#7574). The lines of sight for the channels shown are represented in figure 1 by blue lines.

Figure 6 indicates that the diffusion and inward convection $(V<0)$ are relatively low in the region of $r / a>0.5$, which leads to the fast temporal increase of the soft X-ray signals in the entire SXR channels. In addition, the transport for the on-axis ECH case was almost unaffected by ECH in comparison with the non-ECH case. Therefore, the region in the study was limited to the inner region where $r / a<0.5$. In the region of $r / a=0.4-0.5, D(r)$ was larger than those in other regions, and $V(r)$ was strongly negative, i.e. radially inward, for both non-ECH and on-axis ECH cases. It reproduced the lower signal level of SXR channels 3 and 4 located around the mid-radius region. This strong local inward pinch of Ar 
impurities has also been observed in other tokamaks, regardless of L-or H-modes [12, 24, 27].

(a)
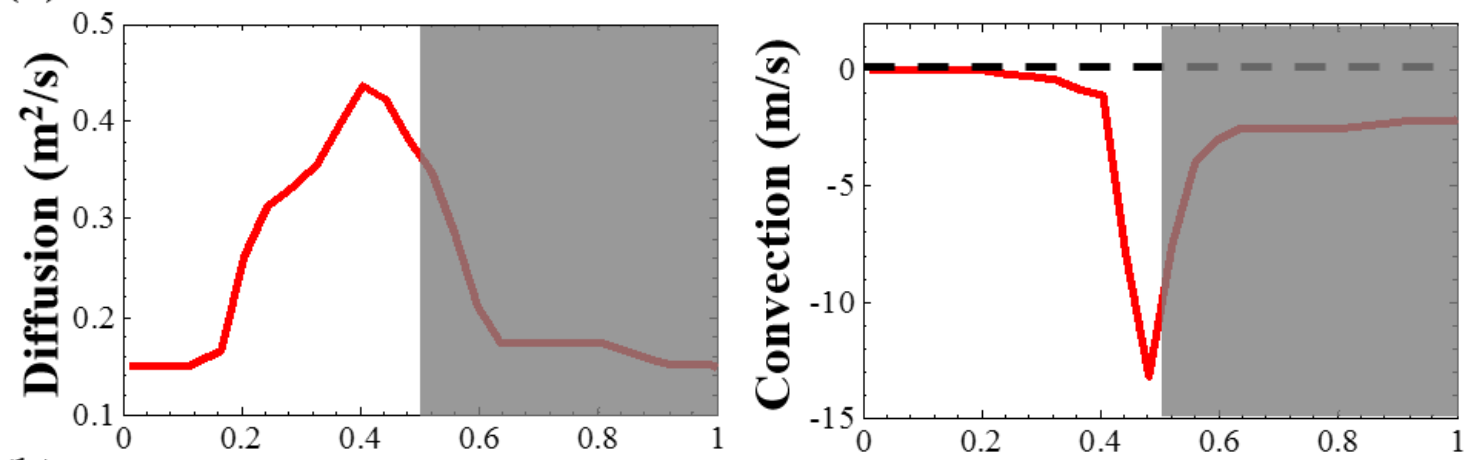

(b)
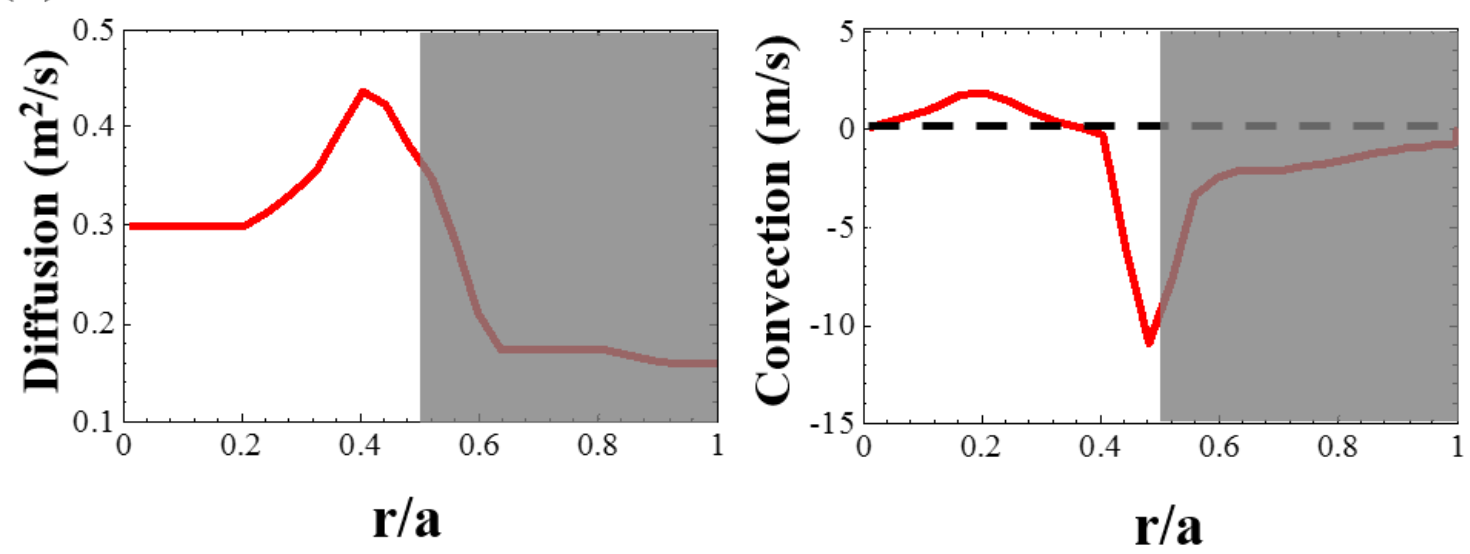

Figure 6. Radial profiles of diffusion and convection coefficients for the (a) non-ECH case and (b) on-axis ECH case. The grey-shaded region denotes the high uncertainty region due to low signal-tonoise ratio of SXR signals.

In the central region where $r / a=0.0-0.3$, both $D$ and $V$ in the ECH case were larger than those in the non-ECH case. The direction of the pinch velocity $V$ reverses from inward $(V<0)$ to outward $(V>$ 0 ). These facts strongly suggest that Ar impurities undergo outward transport more significantly under $\mathrm{ECH}$. These changes are also consistent with the findings of previous impurity transport studies with auxiliary heating [7-13]. Another point to note is that transport coefficients barely change in the region $r / a>0.3$. The effect of the ECH deposition on impurity transport may be local, which was also addressed in ASDEX-U [12]. More discussion on reversed convection with the KSTAR L-mode condition follows in the discussion section.

Figure 7 shows the time evolution of the total Ar density profile and the radial density profiles of each Ar charge state at the peak and stationary times for the non-ECH and ECH cases. These density profiles are calculated by SANCO with the diffusion and convection profiles as shown in figure 6 . In the early stage of the simulations, Ar impurities peak at the edge by gas-puffed influx and then quickly penetrate into the core region as time goes on for both the non-ECH and ECH cases. As seen in figure 7(b), the reversal of the convection velocity produces a hollow Ar density profile in the ECH case. The argon density of $n_{\mathrm{Ar}}=1.1 \times 10^{16} \mathrm{~m}^{-3}$ in the ECH case is smaller than $n_{\mathrm{Ar}}=4.0 \times 10^{16} \mathrm{~m}^{-3}$ in the non-ECH case by a factor of 4 . The main charge states of Ar are He-like (orange curve) and $\mathrm{H}$-like (blue curve). These are consistent with the SXR signals shown in figure 3, which shows weak Ar emission at the core, mainly arising from He-like and $\mathrm{H}$-like charge states. Both the experimental data and the simulations 
clearly indicate the reduction of Ar core accumulation by the on-axis ECH in the KSTAR L-mode plasma.

(a)

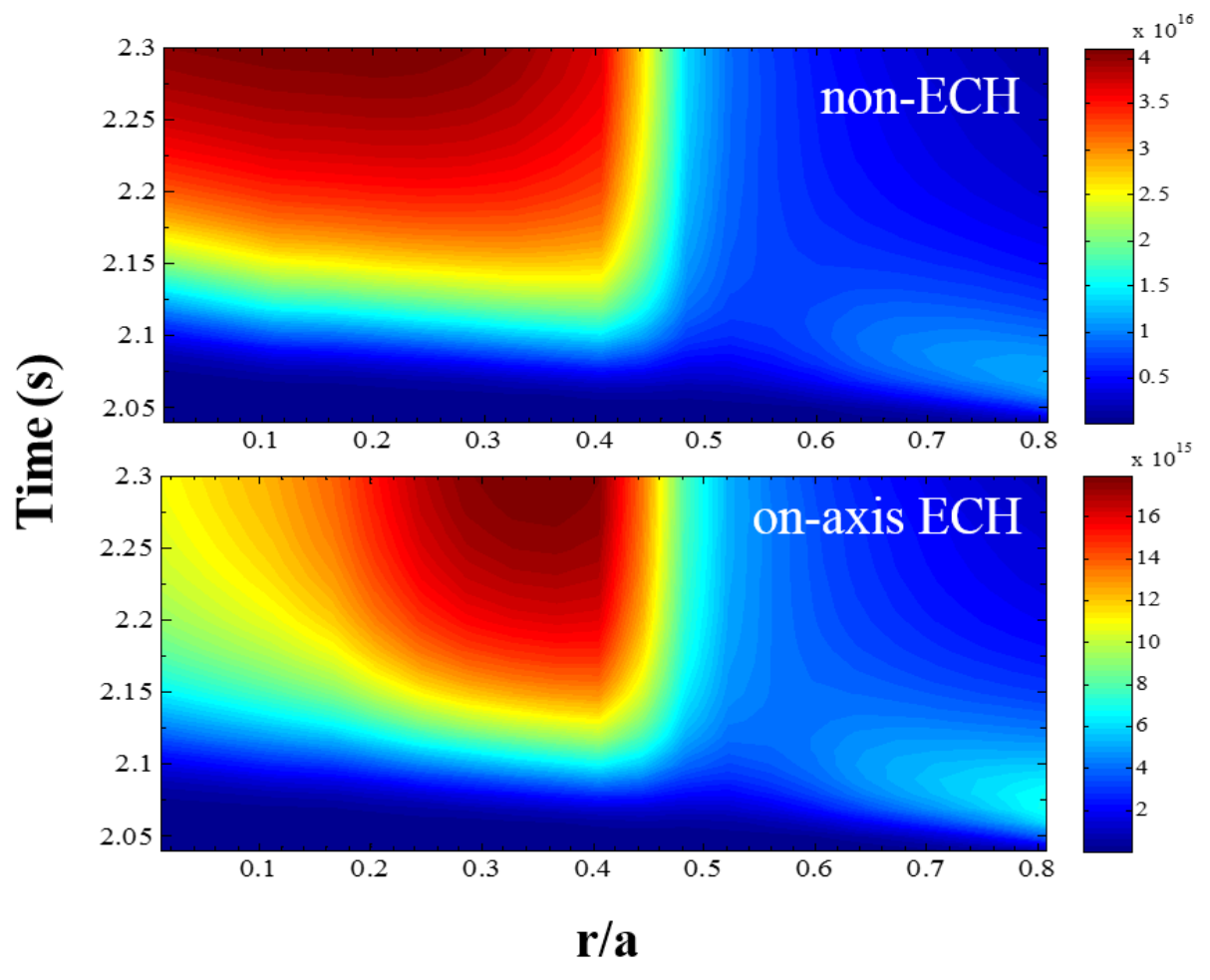

(b)

\section{non-ECH}

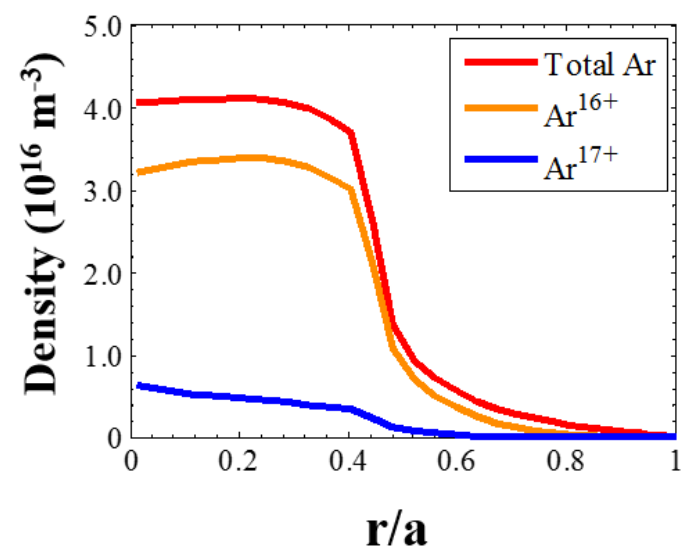

on-axis ECH

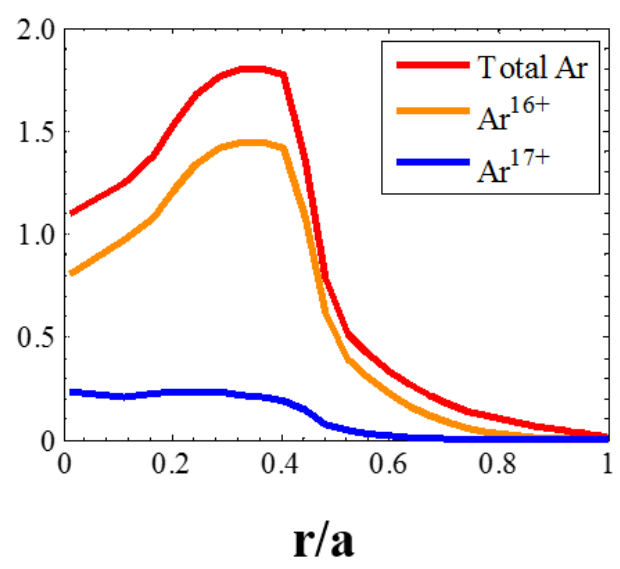

Figure 7. (a) SANCO calculation of the time evolution of radial distribution of total Ar density, and (b) radial Ar density profile of each charge state at peak time for the non-ECH and $\mathrm{ECH}$ cases.

\section{Discussion}

It is known that impurity transport at the core of the L-mode plasmas can be neoclassical $[12,28$, 29] or anomalous [30-32]. Although definitive identification of the impurity transport mechanism in our observation must be deferred to future analysis, a possible scenario for the convection reversal is briefly discussed in this section.

The first step in identifying the impurity transport mechanism would be to estimate neoclassical transport for both the on-axis ECH and non-ECH cases. The neoclassicalAr transport coefficients were 
estimated using NCLASS [33] with the same background plasma profiles used in the SANCO analysis. Plasma rotation was not included in the calculation since the measured core toroidal rotation speed of Ar measured by X-ray imaging crystal spectroscopy (XICS) was about $10 \mathrm{~km} / \mathrm{s}$, which is relatively small. The radial Ar density profiles at the peak time, $2.3 \mathrm{sec}$ in figure 7(b), were applied to the neoclassical transport calculation. The calculated neoclassical transport coefficients are shown in figure 8(a) and (b) for the non-ECH and on-axis ECH cases, respectively. For both cases, the neoclassical diffusivities and convection velocities were smaller by an order of magnitude than the transport coefficients estimated by SANCO. Therefore, neoclassical transport may not be responsible for the experimental observation.

(a)
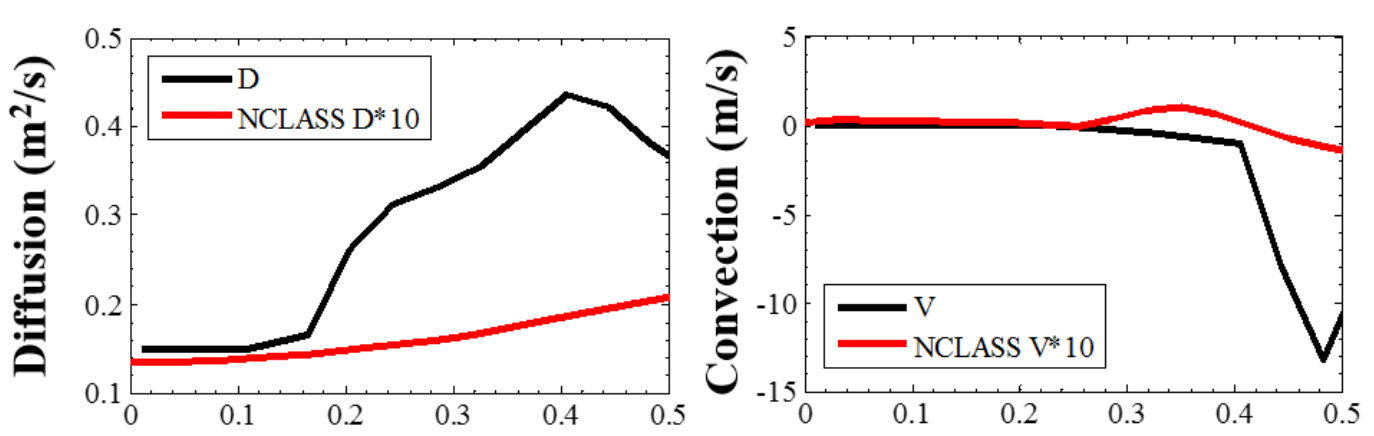

(b)
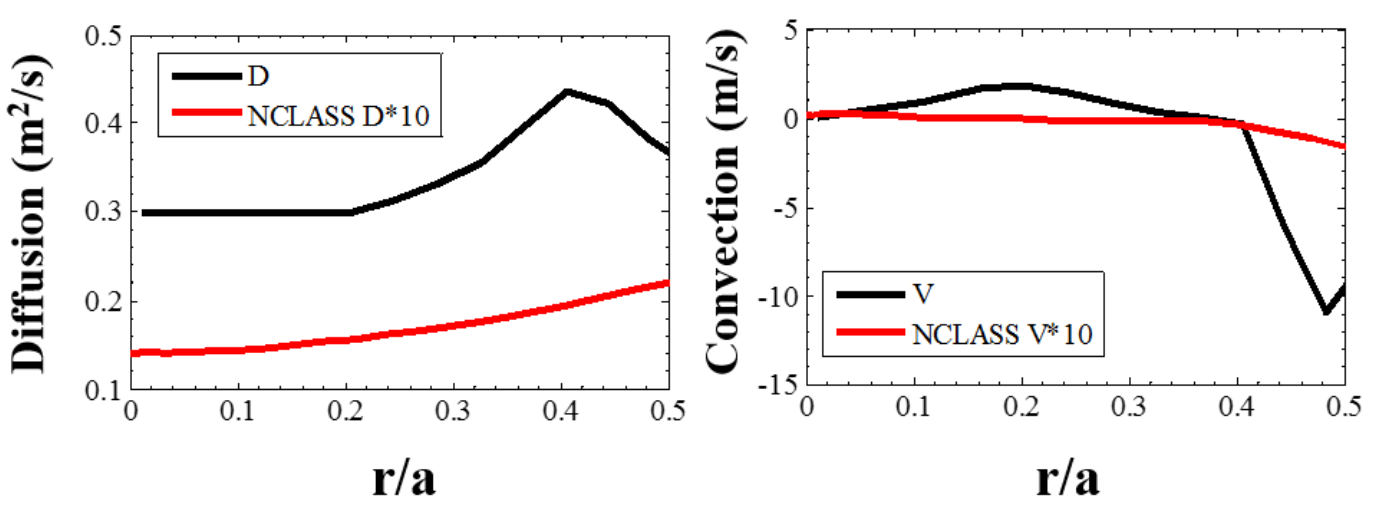

Figure 8. Neoclassical D and V profiles from NCLASS compared with black lines from SANCO calculation for the (a) non-ECH shot (\#7566) and (b) on-axis ECH shot (\#7574). NCLASS results are multiplied by 10 for the sake of easy comparison.

Recently, there have been serious theoretical efforts to investigate the dependence of the direction of impurity convection on ambient micro-turbulence and convection mechanisms [34, 35]. Turbulencedriven transport can indeed change the direction of impurity convection, depending on their convection mechanisms. While the curvature pinch effect tends to drive impurity inward, the thermodiffusion and parallel impurity compression in trapped electron mode (TEM)-driven turbulence can produce inward and outward convection, respectively. On the other hand, the directions of thermodiffusion and parallel impurity compression in ion temperature gradient (ITG)-driven turbulence are opposite to those in TEM, as summarized in table 2.

Table 2. Three types of convective pinch in Weiland multi-fluid model and its direction depending on 
turbulence type [36]

\begin{tabular}{|c|c|c|}
\hline Pinch type & Mechanis m & Pinch direction \\
\hline Curvature pinch & $\begin{array}{l}\text { Compressibility of E x B drift } \\
\text { impurity velocity }\end{array}$ & Inward \\
\hline $\begin{array}{c}\text { Thermodiffusion } \\
\text { pinch }\end{array}$ & $\begin{array}{l}\text { Compression of diamagnetic } \\
\text { drift impurity velocity }\end{array}$ & $\begin{array}{l}\text { Outward (ITG) } \\
\text { Inward (TEM) }\end{array}$ \\
\hline $\begin{array}{l}\text { Parallel impurity } \\
\text { compression }\end{array}$ & $\begin{array}{c}\text { Compression of parallel impurity velocity } \\
\text { fluctuations by fluctuating electrostatic } \\
\text { potential }\end{array}$ & $\begin{array}{c}\text { Inward (ITG) } \\
\text { Outward (TEM) }\end{array}$ \\
\hline
\end{tabular}

The local linear stability analysis of the non-ECH and on-axis ECH shots at the corresponding radial positions, $r / a=0.1,0.2$, and 0.3 with the gradient parameters described in table 3 , over the wavenumber range of $k_{y}=0.1-2.0$, with linear gyrokinetic codes [37-39] showed that the unstable modes were in the electron diamagnetic direction, as shown in figure 9(a). That is, TEM was the dominant instability, and the linear growth rates increased with the on-axis $\mathrm{ECH}$, as shown in figure 9(b), mainly due to larger electron temperature gradients. The linear results, combined with the experimental observation of outward convection with on-axis ECH, seem to suggest that the parallel impurity compression pinch in TEM [14] could play a critical role in the observed reversal of the pinch direction.

Table 3. Gradient parameters for GENE calculation for each shot

\begin{tabular}{ccccc}
\hline Case & $\begin{array}{c}\text { Position } \\
(\mathbf{r} / \mathbf{a})\end{array}$ & $\mathbf{R} / \mathbf{L}_{\mathbf{T e}}$ & $\mathbf{R} / \mathbf{L}_{\mathbf{T i}}$ & $\mathbf{R} / \mathbf{L}_{\mathbf{n e}}$ \\
\hline Non-ECH & 0.1 & 1.13 & 1.44 & 0.45 \\
& 0.2 & 3.45 & 2.59 & 1.18 \\
& 0.3 & 6.60 & 3.83 & 2.13 \\
On-axis ECH & 0.1 & 4.41 & 1.50 & 0.45 \\
& 0.2 & 6.54 & 2.55 & 1.18 \\
& 0.3 & 8.55 & 3.66 & 2.13 \\
\hline
\end{tabular}

Based on the neoclassical calculations and the linear analysis of instabilities for gyroradius-scale turbulence, impurity transport, especially a mechanism for the reversalof convection velocity, is thought to be anomalous, and its characteristics are TEM-like arising from the electron temperature modified by ECH. However, detailed comparison of the experiments with quasi-linear or nonlinear gyrokinetic estimates of impurity transport is beyond the scope of this paper and will be explored in future work. 
(a)

Linear stability (electrostatic)

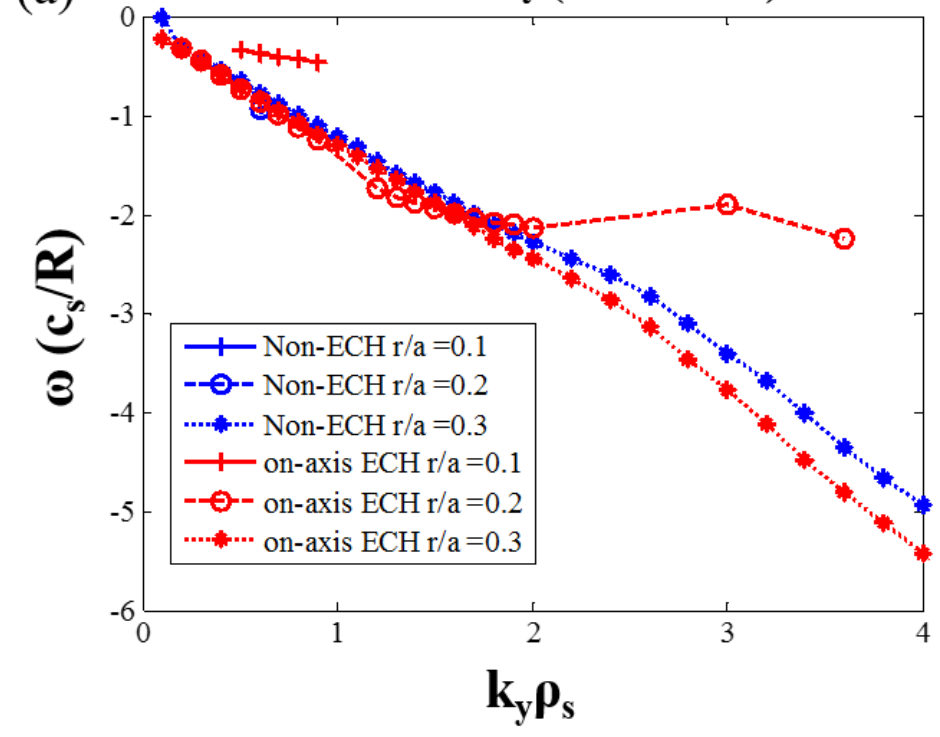

(b)

Linear stability (electrostatic)

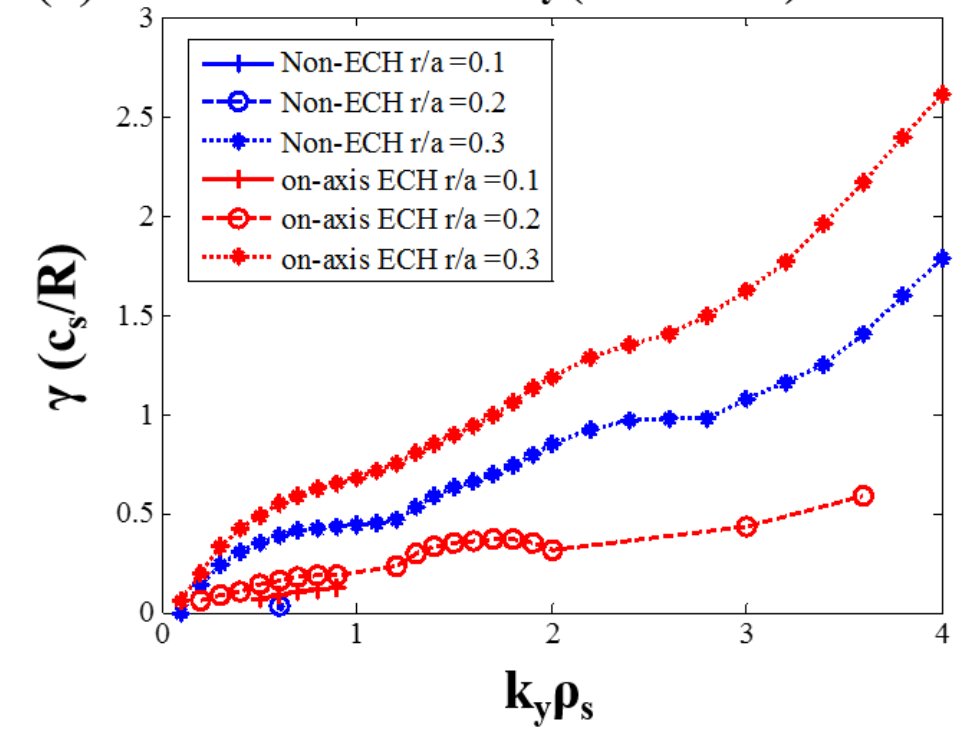

Figure 9. GENE linear stability calculation for the non-ECH and on-axis ECH cases. (a) Linear frequency and (b) linear growth rates. Blue represents the non-ECH case, and red represents the on-axis ECH case. Each solid, dashed, and dotted line indicates a radial position $(r / a=0.1,0.2,0.3)$ respectively.

\section{Summary}

A study on trace Ar impurity transport in KSTAR L-mode plasmas was presented with the emphasis on the reversal of impurity convection direction by on-axis $\mathrm{ECH}$, which produces weak impurity accumulation at the plasma core. Experiments were performed with variation of the heating position of $\mathrm{ECH}$, while measuring the soft X-ray signals of gas-puffed Ar in time. The measured SXR emissivity was lower with ECH than without ECH, particularly in the plasma core. The effect of ECH becomes less significant as the location of ECH deposition is moved away from the magnetic axis. Using the SANCO code, the Ar transport coefficients were calculated from the measured emissivities for non$\mathrm{ECH}$ and on-axis ECH cases. For the ECH case, the reversal of convection from inward to outward was 
observed, and the Ar profiles were estimated to be hollow, as opposed to the peaked Ar profiles and the inward convection observed without ECH. Together with the reduction in the SXR signals, the hollow profiles and the reversal of convection consistently affirm that, in KSTAR L-mode, ECH can reduce impurity accumulation at the core.

Neoclassical calculation using NCLASS and linear gyrokinetic simulation using GENE suggest that the Ar impurity transport in KSTAR L-mode plasma is anomalous rather than neoclassical (the NCLASS results are smaller by $1-2$ orders of magnitude than the transport coefficients estimated by SANCO). The linear gyrokinetic calculations show that ECH increases TEM, which can produce the outward convection of the impurities via parallel compression pinch [14]. This implies that TEM enhancement by ECH may reduce the observed impurity accumulation, although the connection needs further confirmation by nonlinear simulations. Following this study in L-mode, the next step will be to conduct more experiments covering extensive parameters, including $\mathrm{H}$-mode with tungsten as impurities so that experimental ranges and conditions for the effective reduction of core accumulation by auxiliary heating could be mapped for KSTAR, which will be critical for ITER as well.

\section{Acknowledgements}

The first two authors contributed equally to this work. This research was supported by the National R\&D Program through the National Research Foundation of Korea (NRF), funded by the Ministry of Science, ICT (NRF-2014M1A7A1A03045092). One of the authors, Juhyung Kim, acknowledges the support of the World Class Institute (WCI) Program of the National Research Foundation funded by the Ministry of Science, ICT and Future Planning (MSIP) of Korea (WCI 2009-001). The authors are grateful to Prof. S.A. Sabbagh and Dr. Young-Seok Park of Columbia University for providing EFIT magnetic reconstruction data.

\section{References}

[1] Doyle E. J. et al Progress in the ITER Physics Basis Chapter 2: Plasma confinement and transport 2007 Nucl. Fusion 47 S18

[2] Hulse R. et al 1983 Nucl. Technol./Fusion 3259

[3] Hinnov E. et al 1978 Nucl. Fusion 181305

[4] Fussmann G. et al 1991 Plasma Phys. Control. Fusion 331677

[5] Neu R. et al 2002 Plasma Phys. Control. Fusion 44811

[6] Takenaga H. et al 2003 Nucl. Fusion 431235

[7] Puiatti M. E. et al 2002 Plasma Phys. Control. Fusion 441863

[8] Dux R. et al 2003 Plasma Phys. Control. Fusion 451815

[9] Puiatti M. E. et al 2003 Plasma Phys. Control. Fusion 452011

[10] Puiatti M. E. et al 2006 Phys. Plasmas 13042501

[11] Leigheb M. et al 2007 Plasma Phys. Control. Fusion 491897

[12] Sertoli M. et al 2011 Plasma Phys. Control. Fusion 53035024

[13] Valisa M. et al 2011 Nucl. Fusion 51033002

[14] Angioni C. and Peeters A. 2006 Phys. Rev. Lett. 96095003

[15] Angioni C. et al 2007 Plasma Phys. Control. Fusion 492027

[16] Angioni C. et al 2014 Nucl. Fusion 54083028

[17] Casson F. et al 2015 Plasma Phys. Control. Fusion 57014031

[18] Lee S. H. et al 2012 Rev. Sci. Instrum. 83 10E512 
[19] Bogatu I. N. et al 2001 Rev. Sci. Instrum. 721192

[20] Bogatu I. N. et al 2001 43rd American Physical Society Annual Meeting of Division of Plasma Physics, Long Beach, CA, USA, 29 October-2 November 2001; Bull. Am. Phys. Soc. 46226

[21] Bogatu I. N. et al 2002 44th APS Annual Meeting of Division of Plasma Physics, Orlando, FL, USA, 11-15 November 2002; Bull. Am. Phys. Soc. 47, 276

[22] Lauro-Taroni L.et al 1994 21st EPS Conf. on Controlled Fusion and Plasma Physics (Montpellier) p 102 I

[23] Summers H. P. 2004 The ADAS User Manual, version 2.6 http//www.adas.ac.uk

[24] Giroud C. et al 2007 Nucl. Fusion 47313

[25] C.S. Chang, S. Ku, and H. Weitzner 2004 Phys. Plasmas 112649

[26] Whiteford A. 2004 'On the spectral emission of impurity species for diagnostic application to magnetically confined fusion plasmas', Ph.D.Dissertation, University of Strathclyde, Glasgow, U.K.

[27] Greiche A. et al 2009 Plasma Phys. Control. Fusion 51032001

[28] Howard N. et al 2012 Nucl. Fusion 52063002

[29] Scavino E. et al 2003 Plasma Phys. Control. Fusion 451961

[30] Stutman D. et al 2003 Phys. Plasmas 104387

[31] Giannella R. et al 1994 Nucl. Fusion 341185

[32] Guirlet R. et al 2009 Nucl. Fusion 49055007

[33] Houlberg W. et al 1997 Phys. Plasmas 43230

[34] Nordman H. et al 2011 Plasma Phys. Control. Fusion 53105005

[35] Futatani S. et al 2010 Phys. Rev. Lett. 104015003

[36] Giroud C. et al 2007 'Impurity transport at JET On-going analysis from recent campaign', The $13^{\text {th }}$ ITPA Confinement Database \& Modelling Topical Group Meeting,Naka, Japan

[37] Candy J. et al 2003 Phys. Rev. Lett. 91045001

[38] Jenko F. Dorland W., Kotchenreuther M., and Rogers B. N., 2000 Phys. Plasmas 71904

[39] See http://gene.rzg.mpg.de for the code details and access 